\title{
PROPERTIES OF THE WINDS OF T TAURI STARS
}

\author{
NURIA CALVET \\ Centro de Investigaciones de Astronomía, Mérida, Venezuela, \\ and Center for Astrophysics, 160 Concord, MA 02138,USA
}

\begin{abstract}
The properties of Classical T Tauri (TTS) winds - their mass loss rate, temperature, origin, and energetics - are reviewed and interpreted in terms of the most recent observational evidence. TTS winds seem to have their origin in the disk and are powered by accretion. Most TTS have $\dot{M}_{w} \leq 10^{-9} \mathrm{M}_{\odot} \mathrm{yr}^{-1}$ and $\dot{M}_{w} / \dot{M} \sim 0.1$, with $\mathrm{T} \sim 10^{4} K$.
\end{abstract}

\section{Introduction}

From very early on it was recognized that the characteristic blueshifted absorption component in the emission line profiles of T Tauri stars (TTS) indicated the presence of winds (Kuhi 1964). However, the interpretation of the wind and its origin has changed with the understanding of the origin of the activity in TTS. When TTS were thought to posses an amplified solar type kind of activity, winds were expected to be spherically symmetric, emerging from the stellar surface. As it became apparent that the energy source for the activity was accretion energy, and the presence of an accreting circumstellar disk became the natural scenario, the evidence pointed to the disk as the source of material and power in the wind. Two important points have arisen in this latest picture. First, the wind is far from spherically symmetric, at least at small spatial scales. Second, emission lines as a whole should no longer be seen as wind indicators, i.e., the bulk of the line probably arises in the magnetospheric infalling flow joining the disk and the star (see Edwards, this volume), and do not trace the outflowing material.

In this review, I will refer mostly to the properties of mass ejected near the star, at scales smaller than $100 \mathrm{AU}$, as inferred from present day observations, and discuss the observational constraints that theoretical models should explain to be applicable to TTS winds. In section 2, I discuss the 

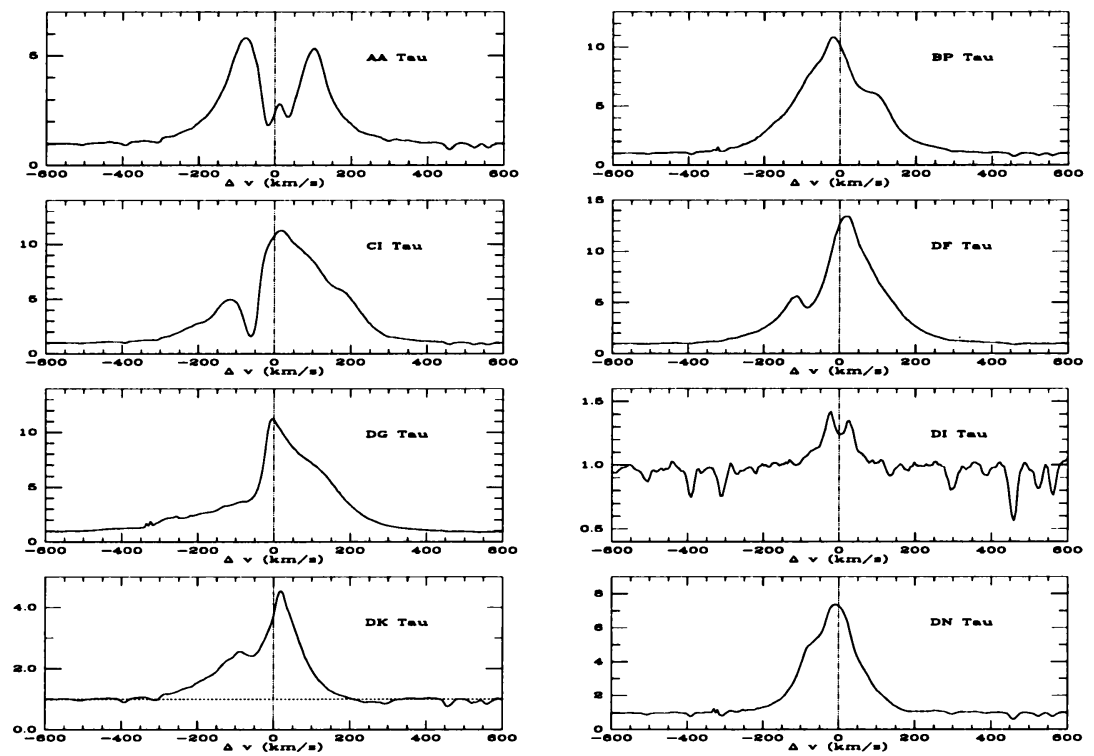

Figure 1. Typical H $\alpha$ line profiles of TTS

appropriate wind indicators within the present picture of TTS. In the following sections, I review the region of formation (section 3), and the physical conditions of the wind (section 4) based on the information provided by those indicators. I will refer only to the Classical T Tauri stars (CTTS), and call them TTS. The weak line T Tauri stars show no indication of the presence of a wind, at least of the same nature as that of CTTS (Hartigan, Edwards, \& Ghandour 1995, HEG hereafter), and will not be discussed.

\section{Wind Indicators. Separating Magnetosphere and Wind}

Figure 1 shows $\mathrm{H} \alpha$ line profiles for a sample of TTS. The typical features of the line profiles are: (1) An emission peak at nearly zero velocities; (2) A blueshifted absorption component (BAC), at velocities $\sim-50-150 \mathrm{~km} \mathrm{~s}^{-1}$. A few stars, like AA Tau, show a strong central absorption, which is yet to be explained; (3) Extended blue and red wings, to velocities up to $\pm 250 \mathrm{~km} \mathrm{~s}^{-1}$. Stars without accretion, as the WTTS DI Tau in Figure 1, show a narrow line profile.

Original interpretations sought to explain the whole emission line profile as produced in the wind. Spherical wind models (Hartmann, Edwards, \& Avrett 1982; Lago 1984, 1986; Natta, Giovanardi, \& Palla 1988; Hartmann etal 1990; Giovanardi etal 1991; Johns \& Basri 1995a) can successfully 
explain the fluxes of emission lines, but generally fail in explaining the line profiles. As shown in Calvet, Hartmann, \& Hewett (1992, their figure 3) profiles from spherical winds are typically $\mathrm{P}$ Cygni type, with deep, below the continuum, BACs and redshifted emission peaks, unlike the observed profiles (c.f. Figure 1). The stochastic spherical wind of Mitskevich, Natta, \& Grinin (1993) can explain the observed line profiles, basically because these authors assumed that the wind decelerated outwards. However, it is very difficult to find theoretically a hydrodynamic wind solution with this property (Holzer etal 1983). Spherical winds also fail in explaining the decreasing depth of the BAC going up the Balmer series. Generally, in wind models, the source function falls steeply in the outer regions relative to the Planck function, and this effect gets stronger for lower opacity lines. As a result, the depth of the BAC increases for higher series lines, contrary to the observations.

Given the failure to explain the observed emission line profiles with classical winds, other alternatives were explored. Since the middle $80 \mathrm{~s}$, it is generally accepted that TTS are surrounded by disks responsible for the infrared excess, a model originally proposed by Lynden-Bell and Pringle (1974). In this picture, the disk material joined the star through a narrow boundary layer, where it slowed down from Keplerian to the slow rotation of the stellar surface. Nearly half of the accretion energy was supposedly dissipated in this layer; turbulent motions [and shocks] were expected, and winds could potentially be produced (Pringle 1989; Bertout \& Regev 1992). Calvet, Hartmann, \& Hewett (1992) explored the feasibility of this model by calculating line profiles from a configuration near the star with characteristics appropriate to boundary layers. The resultant line profiles still could not reproduce the observed profiles (c.f. Figure 3 of Calvet, Hartmann, \& Hewett [1992]). They were too broad, due to rotation, the peak of the emission was still too redshifted, and at typical inclinations to the line of sight, the BAC was too deep.

In recent years, it became apparent that, given the expected magnetic field strengths in the stellar surface ( few KG; Basri, Marcy, \& Valenti 1992; Guenther, this volume), and typical mass accretion rates ( 10 $10^{-8} \mathrm{M}_{\odot} \mathrm{yr}^{-1}$, Gullbring etal 1997), the inner disks of TTS would be disrupted by the magnetic field and matter will move into the star along the field lines in nearly free fall (Bertout, Basri, \& Bouvier 1988; Krautter, Appenzeller, Jankovics, 1990; Königl 1991; Calvet \& Hartmann 1992; Shu etal 1994).

One of the most important evidences in favor of the magnetospheric model is that this model can successfully reproduce the observed profiles of emission lines. In the magnetospheric flow, the bulk of the emission comes from material moving at low velocities far from the star, while the wings 

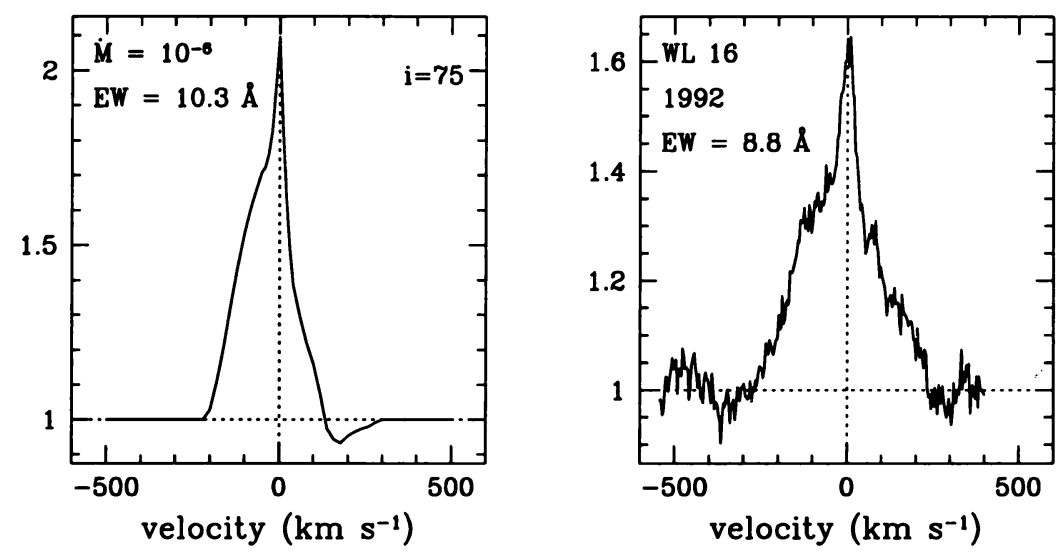

Figure 2. $\operatorname{Br} \gamma$ line profiles. Left: magnetospheric profile from Muzerolle etal (1997). Right: profile of WL 16 from Najita etal (1996)

are produced by the material close to the stellar surface, moving at the free-fall velocity. Therefore, the peak at nearly zero velocity and the line width are naturally explained. Magnetospheric profiles can explain very well the main features of Balmer lines (Hartmann, Hewett, \& Calvet 1994). The multilevel, extended Sobolev radiative transfer in the magnetospheric flow by Muzerolle etal (1997) has extended this early treatment to higher Hydrogen series. Figure 2 compares the observed $\mathrm{Br} \gamma$ line profile for WL16 (Najita etal 1996) with a magnetospheric profile of Muzerolle etal (1997). The magnetospheric profile reproduces very well the main features of the observed profile.

The success of the magnetospheric model in explaining the observed line profiles has important consequences. First, the bulk of the line flux comes from the infalling, magnetospheric material, and it is not formed in the wind. Therefore, the hydrogen emission line fluxes are not indicators of the wind. The only true wind signature in permitted lines is the blueshifted absorption, which cannot be explained in terms of the magnetospheric model. Second, all the mass loss rate estimated based on the flux of the hydrogen emission lines, both optical and infrared lines (Natta etal 1988; Hartmann etal 1990) are meaningless, since those lines do not arise in the outflowing region.

Only analysis of the BAC can render an estimate of the rate of mass loss in TTS from permitted lines. On the other hand, the low excitation forbidden lines seen in TTS (Hamann 1994; HEG) do probe the outflowing material; in the cases where a spatially extended jet has been resolved, 


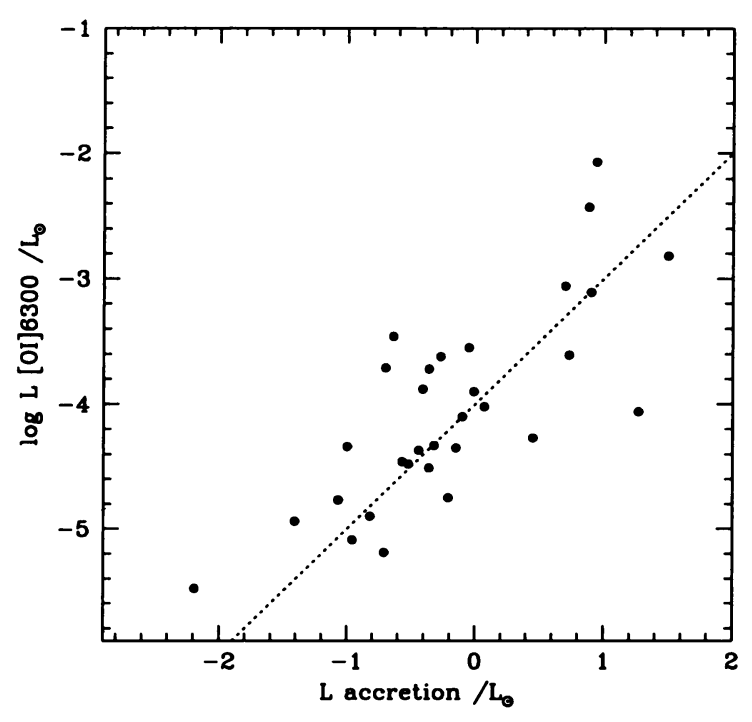

Figure 3. Total luminosity in [OI] 6300 vs. accretion luminosity, from data in HEG

the forbidden emission at the stellar position merges smoothly with the jet emission (Solf \& Böhm 1993; Hirth, Mundt, \& Solf 1993). Analysis of the forbidden lines provides the best available estimates so far of the mass loss rate in TTS.

\section{Origin of the Wind}

Since most of the flux of the permitted emission lines comes from the magnetospheric flow, they are no longer a tool to infer the physical characteristics of the wind. The forbidden lines provide the required information at large scales, but probably will be quenched in the densest, innermost zone, and we only have the profile of the BAC to analyze this region. Nonetheless, the analysis of these observational indicators provides clues to discern between the different models that have been discussed for the region where the wind is ejected and powered.

A wind is present whenever the disk is present. Stars that have near infrared excess have forbidden [OI] emission, while stars without excess do not show forbidden lines (Cabrit etal 1990; HEG). A near infrared excess is evidence that a disk with a range of temperatures (in contrast to the single temperature stellar flux) is present (Hartmann, this volume, and 
references therein), but a correlation with the infrared excess only proves that the disk presence is an essential ingredient for the existence of the wind, because the disk can be heated externally by stellar irradiation (Kenyon \& Hartmann 1987; Calvet etal 1992). However, the correlation between the forbidden line luminosity and the accretion luminosity, as measured from the veiling of absorption lines (HEG), shown in Figure 3 strongly suggests that in addition to having a disk origin, TTS winds are powered by accretion energy. The form into which accretion energy is transmitted to the wind in TTS is still a question of debate.

\subsection{THE FU ORI WIND}

The case of the FU Ori objects can give some insight into the origin of the wind of the less energetic TTS. FU Ori objects are thought to be high mass accretion disks that surround a low mass star (Hartmann \& Kenyon 1996; Hartmann, this volume). In one case, a strong line CTTS was observed previous to the disk outburst, so by looking at FU Ori we are in principle observing the same physical system, subject to a higher release of potential energy.

FU Ori objects prove to be the best clear case of a disk wind, in that we can see the wind accelerating region. The energy released by viscous processes in FU Ori disks heats the inner disk regions to temperatures $\sim 7000 \mathrm{~K}$, so the spectrum of the disk atmosphere shows metallic absorption lines with a wide range of strengths. The weak lines are formed deeply in the disk atmosphere, in a region where the Keplerian rotational velocity is larger than the slow expansion velocity, so their line profile shows the characteristic double peak structure expected from a rotating disk. On the other hand, the strong lines are formed high up in the atmosphere, where the expansion velocity is larger than the rotational velocity; their line profiles are single and blueshifted (Calvet etal 1993). The upper panel Figure 4 shows the result of detailed modeling of metallic lines of differing strengths for the case of FU Ori, from Hartmann \& Calvet (1995). As the strength of the line increases, the peaks of the double profile merge into one. The lower panel shows observed positions of the peaks of absorption lines in the spectrum of FU Ori (also reported by Petrov \& Herbig [1992]). The observations behave as predicted by a rotating accelerating disk wind.

A mass loss rate $\dot{M}$ of $\sim 10^{-5} \mathrm{M}_{\odot} \mathrm{yr}^{-1}$ is found for FU Ori (Croswell, Hartmann, \& Avrett 1987; Calvet etal 1993), yielding a ratio $\dot{M}_{w} / \dot{M} \sim 0.1$.

\subsection{THE REGION OF ORIGIN OF TTS WINDS}

Figure 5 shows observations of the $\mathrm{H} \alpha$ and $\mathrm{Na}$ I D lines for a sample of young stellar objects arranged from top to bottom in decreasing order 

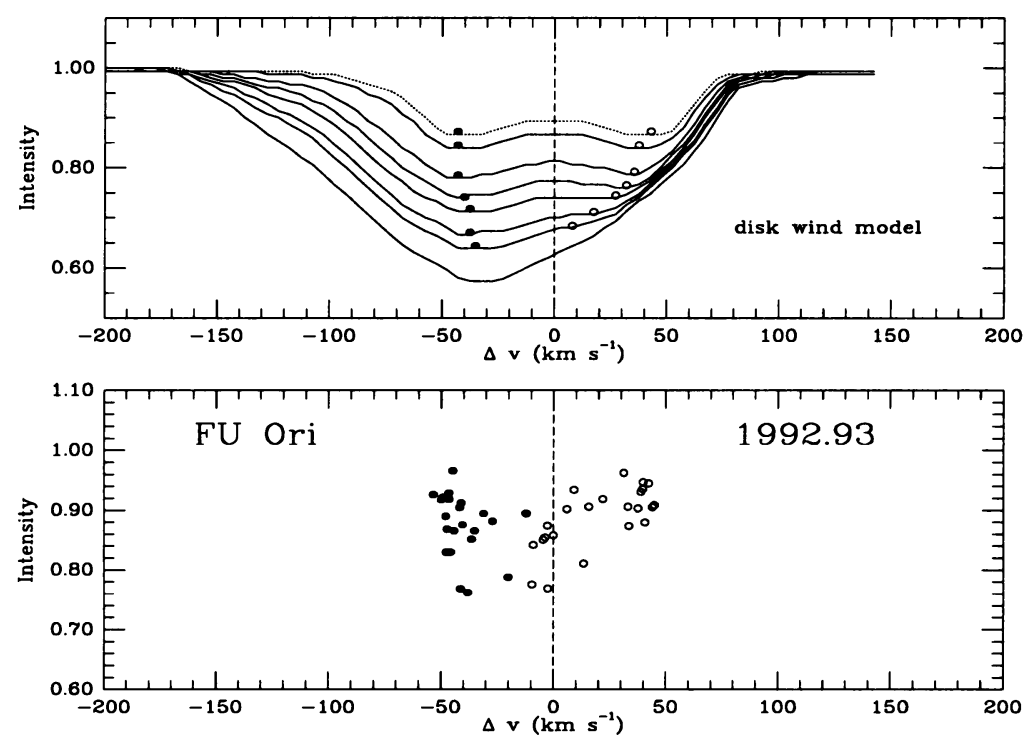

Figure 4. Line profiles for FU Ori disk wind (from Hartmann \& Calvet 1995)

of mass accretion rate. At the top, the lines in FU Ori show very strong blueshifted absorption. As the mass accretion rate in the disk decreases, the lines develop emission, probably associated with the emergence of the magnetosphere, which is thought to be crushed out by the rapid accretion rates in FU Ori (Hartmann 1997). At the same time, the strength of the BAC decreases appreciably, to the extent that it is almost absent in the $\mathrm{Na} I \mathrm{D}$ lines of the lowest mass accretion rate TTS.

Although the profiles shown in Figure 5 have been chosen to show the maximum effect, they agree with the previous conclusion that the winds are powered by accretion, in the sense that they are stronger in the highest mass accretion rate objects. Similarly, since the BAC has to be formed very near the star to absorb the stellar and magnetospheric background light, the profiles shown indicate that the density in the inner accelerating wind region decreases with mass accretion rate.

From the observations of individual BAC, however, it is difficult to deduce the exact region of formation of the wind. Two possibilities have been advanced so far. The wind may arise in the inner disk region, similarly to the case of the FU Ori objects ( Blandford \& Payne 1982; Pudritz \& Norman 1983; Königl 1989; Camenzind 1990; Pelletier \& Pudritz 1992; Gómez de Castro \& Pudritz 1993; Safier 1993; Paatz \& Camenzind 1996). 

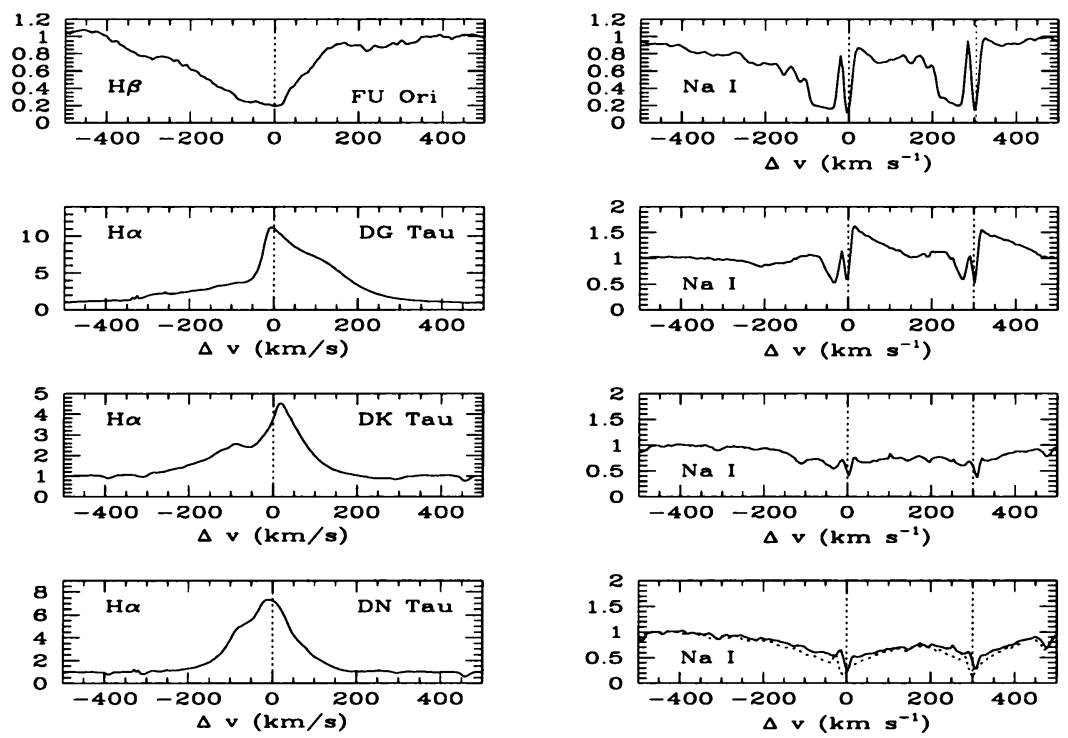

Figure 5. Line profiles of $\mathrm{H} \alpha$ and $\mathrm{Na} \mathrm{I} \mathrm{D}$ for objects arranged by mass accretion rate. From top to bottom: FU Ori, $\dot{M} \sim 10^{-4} \mathrm{M}_{\odot} \mathrm{yr}^{-1}$, DG Tau, $\dot{M} \sim 2 \times 10^{-6} \mathrm{M}_{\odot} \mathrm{yr}^{-1}$, DK Tau, $\dot{M} \sim 4 \times 10^{-7} \mathrm{M}_{\odot} \mathrm{yr}^{-1}$, DN Tau $\dot{M} \sim 3 \times 10^{-8} \mathrm{M}_{\odot} \mathrm{yr}^{-1}$. HEG data. The sharp lines at 0 velocity in the $\mathrm{Na} I \mathrm{D}$ profiles are of interstellar origin.

Alternatively, the wind may be produced at the same point where the magnetospheric flow is launched, at the radius where the disk corotates with the star, as envisaged by the X-wind model (Shu etal 1994; Najita \& Shu 1994; Shu etal 1995). It has proven difficult to device observational tests to distinguish between both kinds of models, especially because the origin of the heating and therefore, the temperature structure, are not well known.

However, variability analyses of line profiles with time baselines covering significantly several stellar periods have provided strong clues as to the location of the wind formation region. The time series analyses comprise several aspects: (a) Search for periodicities in different parts of the line profiles, and compare them with the stellar period; (b) Determine characteristic time scales for variability; (c) Search for correlations between the variations in different parts of the line profiles, to indicate relationships between the physical regions where each arise.

A long time baseline of 37 nights with hundreds of spectra has provided important indications for the characteristics of the BAC and thus of the wind (Johns etal 1992; Giampapa etal 1993; Johns \& Basri 1995a,b; 
Johns-Krull \& Basri 1997). In a sample of 7 stars where the profiles of the strong emission lines have been monitored, the BAC has been found to vary periodically with the same period as the star in one case. This observation supports the view that the wind comes from the corotation radius, in agreement with the predictions of the $\mathrm{X}$-wind model. However, in the rest of the sample, six stars, the BAC is uncorrelated with other parts of the line profile; it is either not periodical, or if periodical, does not vary with the stellar period. Moreover, it shows a longer variation time scale than the rest of the line profile. The authors conclude from these observations that (1) the wind formation region is detached from the region of formation of the main emission, and (2) the wind formation region is exterior to the formation of the main emission. This evidence supports the view that the wind is produced in the disk, in a region exterior to the magnetosphere.

Since the one star where the BAC varies with the stellar period is not a representative TTS, in that it has a higher mass and few evidences of the TTS activity (Edwards, this volume), while the rest of the sample consists of more typical TTS, the variability studies carried out so far indicate a disk origin for the T Tauri wind. It is important, however, to increase the sample of TTS with significant time series coverage to confirm this indication.

\subsection{FORBIDDEN LINES. EXTENDED REGION}

The forbidden lines probe the extended, lower density regions of the wind. However, the information they provide on the geometry of the wind region is not yet conclusive.

The line profiles of forbidden lines in TTS are generally blueshifted. The overall blueshift of the line has been interpreted as indicative of the presence of a disk, which hides the receding flow of the line (Jankovics, Appenzeller, \& Krautter 1983; Appenzeller, Jankovics, \& Östreicher 1984; Edwards etal 1987). In addition, the line profiles of forbidden lines in TTS are often double peaked. Following HEG nomenclature, the peak at velocities $\geq-60 \mathrm{~km} \mathrm{~s}^{-1}$ is called the low velocity component, LVC, while the peak at larger negative velocities is referred to as the high velocity component, HVC.

Figure 6 shows the number of stars where the LVC or the HVC peak is present in the HEG sample, arranged in order of mass accretion rate. The HVC is clearly present only in the high mass accretion stars, while the low velocity component is more ubiquitous. Line ratios indicate that the excitation conditions for both components in a given star are different, with a higher density in the LVC (Hamann 1994; HEG; Solf, this volume). In addition, the HVC is generally spatially resolved, and extends into jets when they can be seen (Solf \& Böhm 1993; Hirth, Mundt, \& Solf 1993; 

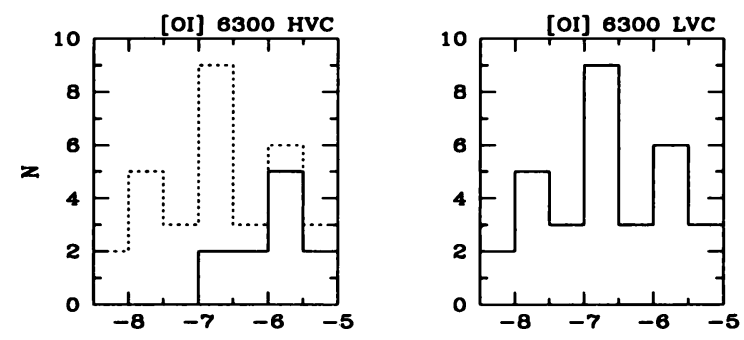

Log Mass Accretion rate

Figure 6. Number of stars with given mass accretion rate where a LVC peak (right panel) or a HVC peak (left panel) is present in [OI] 6300. The dotted line is the total number of stars in the sample, for comparison. Data from HEG

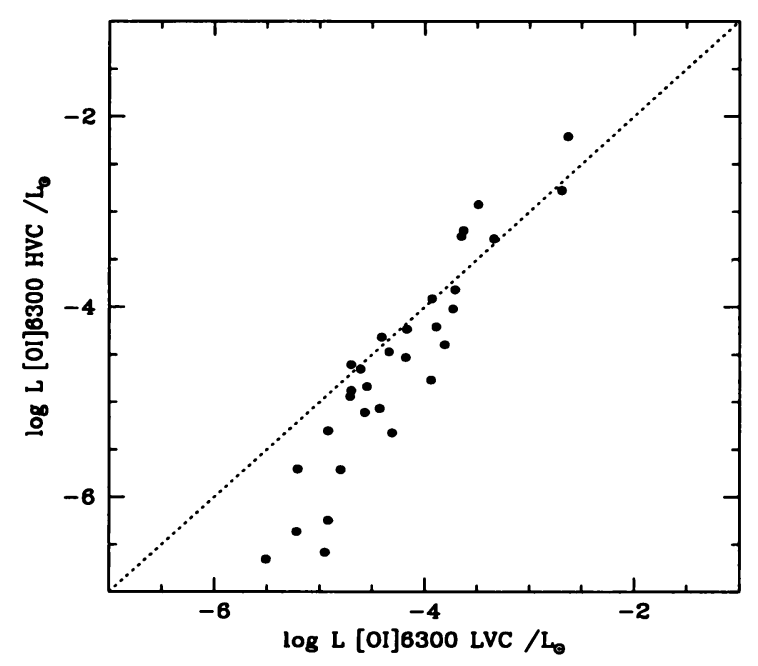

Figure 7. Luminosity of the HVC vs luminosity of the LVC of [OI] 6300. Data from HEG.

Hirth etal 1994). The LVC in general is not resolved, except in [SII] in a few cases (Solf \& Böhm 1993).

These observations have led to the suggestion that the two components arise in two distinct physical regions, in contrast to the models that propose geometric or/and inclination effects to explain the profiles (Edwards 
etal 1987; Raga 1989; Safier 1993; Hartmann \& Raymond 1989; Ouyed \& Pudritz 1994). In this view, the HVC would correspond to the jet itself while the LVC would come from a wind arising in outer disk regions where the escape velocity is low (Kwan \& Tademaru 1988; 1995). However, as shown in Figure 7, the luminosities of the two components are tightly correlated. Moreover, the slope of the correlation is greater than one, suggesting a density effect: at low luminosities, only the higher density low velocity component is present; at high luminosities the wind overall has higher densities, and the low velocity component, produced in the densest regions, may be quenched. This behavior is in agreement with the suggestion that there is only one main emitting region, seen at different scales (Camenzind, this volume). The issue of the origin of the forbidden lines is still an open question.

\section{Mass Loss Rates}

The determination of mass loss rates in the winds of TTS requires the knowledge of the excitation conditions in the emitting region, as well as of its geometry. As these quantities are very uncertain, mass loss rate determinations are only order of magnitude estimates. In this section, I discuss the mass loss rates from the study of the forbidden lines. In addition, I use the BAC of lines with differing ionization and excitation characteristics to constrain the density and temperature of the inner accelerating region of the wind. With these quantities, I derive crude estimates of the mass loss rate from the permitted lines and find them to be consistent with the forbidden line results. Again, these estimates are only appropriate to order of magnitude, and detailed analysis of simultaneous spectra of individual stars should be made to obtain more accuracy.

\subsection{FORBIDDEN LINES}

Mass loss rate estimates of TTS have been obtain by HEG. They use only the HVC of [OI] 6300 for their calculations, since this is the component clearly associated with the jet. They include as HVC any emission at large negative velocities, even if there is no line maximum associated with it. They also assume that the forbidden emission is produced in a shock.

The line luminosity is given by

$$
L_{l} \simeq n_{u} A_{u l} h \nu V
$$

where $n_{u}$ is the upper level of the transition producing the line of frequency $\nu, A_{u l}$ is the Einstein coefficient, and $V$ is the emitting volume.

At the same time, the total emitting mass is 


$$
M \simeq \mu m_{H} n_{H} V
$$

where $\mu$ os the mean atomic weight, and $n_{H}$ is the total hydrogen density. The usual symbols are used for other atomic constants.

If the observations are limited to a region of size $l$, in which the material is moving at velocity $v$, then the mass loss rate can be estimated as

$$
\dot{M}_{w} \simeq M \frac{v}{l}
$$

Combining these equations, we get a relationship between the mass loss rate and the line luminosity,

$$
\dot{M}_{w} \simeq \mu m_{H}\left(\frac{v}{l}\right)\left(\frac{n_{H}}{n_{E}}\right)\left(\frac{n_{E}}{n_{E} i}\right)\left(\frac{n_{E}^{i}}{n_{u}}\right) L_{l}
$$

where $n_{E}$ is the total density of the element producing the line and $n_{E}{ }^{i}$ is the density of this element in the corresponding ionization stage. To evaluate this expression, HEG take $v$ from the observed velocity shift and $l$ from the size of the slit. The abundance of the element gives $n_{H} / n_{E}$. To evaluate the ionization stage, $n_{E} / n_{E}{ }^{i}$ and the relative population of level $u$, $n_{E}{ }^{i} / n_{u}$, knowledge of the temperature and the electron density is required. HEG assume a temperature of $8000 \mathrm{~K}$, arguing that this is the temperature where OI emits the most in shocks. They also assume that most of the oxygen is neutral and most of the sulphur once ionized. To estimate the electron density, they use the ratio of the HVC fluxes of [S II] 6731 and [OI] 6300, and a two level atom formulation.

With these assumptions HEG analyze a sample of 31 stars with accretion, of which 25 have measurable high velocity emission in [OI], for which they obtain mass loss rates in the range between $10^{-10} \mathrm{M}_{\odot} \mathrm{yr}^{-1}$ and $3 \times 10^{-7} \mathrm{M}_{\odot} \mathrm{yr}^{-1}$, with a median value of $\sim 10^{-9} \mathrm{M}_{\odot} \mathrm{yr}^{-1}$. In the case of RW Aur, HEG derive $\dot{M}_{w} \sim 3 \times 10^{-8} \mathrm{M}_{\odot} \mathrm{yr}^{-1}$, in good agreement with the independent determination of mass loss rate from direct analysis of the resolved jet (Bacciotti, Hirth, \& Natta 1996).

The mass loss rate increases with mass accretion rate. Using the mass accretion rate determined by HEG, $\dot{M}_{w} / \dot{M}$ is $\leq 10^{-2}$. However, more recent determinations of mass accretion rate seem to indicate that the HEG mass accretion rates are overestimated by a factor $\sim 10$ (Gullbring etal 1997, Hartmann, this volume). If this is the case, then $\dot{M}_{w} / \dot{M} \sim 0.1$ is a more typical value. 


\subsection{PERMITTED LINES}

Analysis of the BAC of emission lines is difficult because there are many parameters involved: temperature and density, velocity field, geometry, and inclination. Theoretical models give indication on some of these parameters, like the geometry and the velocity field. However, both the basic uncertainty in the excitation characteristics in the theoretical models and the lack of reliable inclination estimates for individual stars make comparison with observations difficult.

However, even with these uncertainties, order of magnitude estimates can be done. The BAC has to form within a few stellar radii from the star, since the atoms producing it have to absorb the stellar plus magnetospheric background. We can safely assume that this may still be the accelerating region of the flow, so that we can use the Sobolev approximation to estimate the optical depth at velocity $v$ :

$$
\tau_{v}=\frac{\pi e^{2}}{m c} \frac{f c}{\nu_{o}} \frac{n_{l}(v)}{d v / d z}
$$

where $f$ is the oscillator strength of the line with center frequency $\nu_{o}, n_{l}(v)$ is the density of the lower level of the transition of atoms moving at velocity $v$, and $d v / d t$ is the acceleration of the region moving at that velocity.

The mass loss rate can be estimated as

$$
\dot{M}_{w} \simeq \Delta A v \mu m_{H} n_{H}(v)
$$

where $\Delta A$ is the area crossed by the atoms moving at $v$. Since $n_{l}(v) / n_{H}(v) \sim$ $n_{l} / n_{H}$ is a function of $n_{H}$ and $\mathrm{T}$, if we have an estimate of $\Delta A$ and $v$, we can calculate the optical depth of a given line at that velocity as a function of $\dot{M}_{w}$ and T, using these two expressions. As the absorption occurs very close to the star, we take $\Delta A \sim \pi\left(2 R_{*}\right)^{2}$, and with typical velocities and radii, $v \sim 100 \mathrm{~km} \mathrm{~s}^{-1}$ and $d v / d z \sim 100 \mathrm{~km} \mathrm{~s}^{-1} / \mathrm{R}_{*}$.

With this procedure, we can calculate optical depths at the center of the $\mathrm{BAC}$, and compare with observations to determine the physical conditions of the region producing the BAC. Several observations can be used: (a) $\tau(H \alpha) \sim 1-10$. Natta (1996) measured the optical depth at the center of the BAC in $H \alpha$ for a range of TTS with differing mass accretion rates. She found it to be of order 1 or less, except in the most energetic objects; (b) $\tau(B r \gamma)<<1$. Najita, Carr, \& Tokunaga (1996) observed line profiles of $\operatorname{Br}(\gamma)$ for a sample of TTS, generally of high mass accretion rate. They found no hint of the presence of a BAC, even in objects that show it very strongly in $\mathrm{H} \alpha$; (c) $\tau(\mathrm{Pa} \beta)<<1$. Folha etal (1997) find that $\mathrm{Pa} \beta$ does not show the BAC either, except possibly in one case of a star, CW Tau, which has a high mass loss rate as determined from the forbidden lines. 


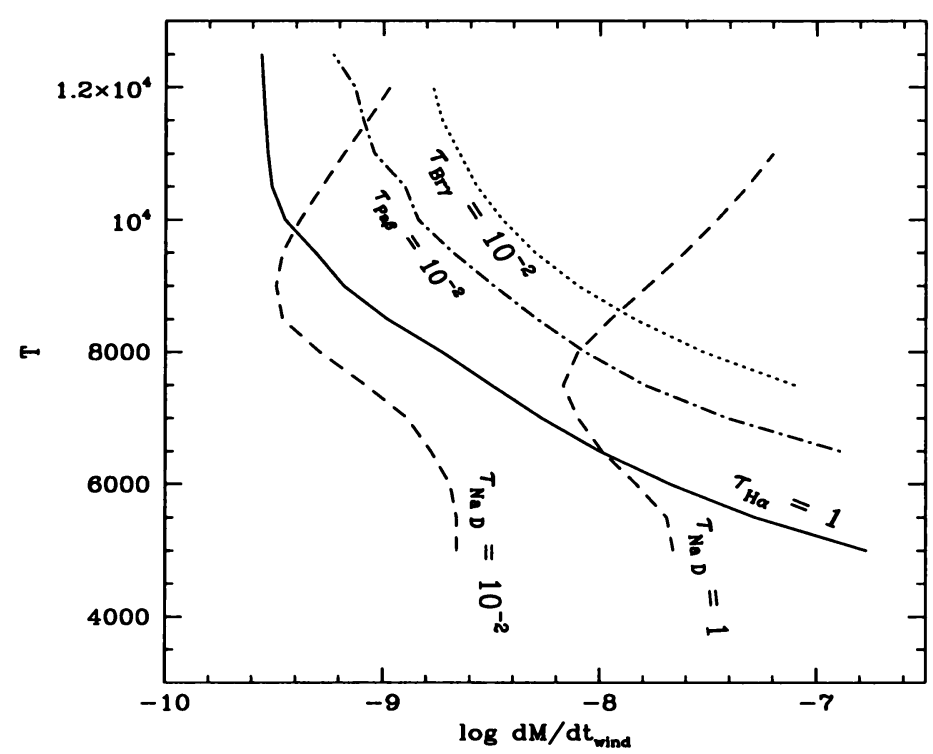

Figure 8. $\dot{M}_{w}$ vs. T diagram with lines indicating typical values of the optical depth of $\mathrm{H} \alpha, \mathrm{Br} \gamma, \mathrm{Pa} \beta$, and $\mathrm{Na} I \mathrm{D}$.

(d) $\tau(N a I D)<<1$ for most $\mathrm{T}$ Tauri stars, of order 1 for the high mass accretion rate objects; (c.f. spectra in Edwards etal 1994). These constraints can be incorporated in a $\left(\dot{M}_{w}, \mathrm{~T}\right)$ diagram as shown in Figure 8. $\tau \sim 10^{-2}$, which corresponds to $\sim 1 \%$ of the neighboring flux, has been adopted as an upper limit when the absorption is not seen.

As noted by Natta \& Giovanardi (1990), the Na I D lines are good indicators of density. Objects which show Na BAC have $\dot{M}_{w} \sim$ few $\times 10^{-8} \mathrm{M}_{\odot} \mathrm{yr}^{-1}$, while for objects that do not show Na I absorption, $\dot{M}_{w} \leq 10^{-9} \mathrm{M}_{\odot} \mathrm{yr}^{-1}$. The hydrogen lines, on the other hand, are good indicators of temperature. In particular, the absence of absorption in the infrared lines, especially $\mathrm{Pa} \beta$, puts strong constraints on the temperature of the absorbing region. The temperature has to decrease as $\dot{M}_{w}$ increases, in agreement with the low values of $\tau(H \alpha)$, since a lower temperature is required to have $\tau H(\alpha) \sim 1$. This behavior is expected, since radiative cooling increases with density (c.f. Hartmann, Edwards, \& Avrett 1982). For the high $\dot{M}_{w}$ objects, which show strong BACs in $\mathrm{H} \alpha$ and $\mathrm{Na}$ I D, the temperature of the absorbing region has to be lower than $\sim 6000 \mathrm{~K}$. In contrast, for the lower $\dot{M}_{w}$ objects to show $\mathrm{BAC}$ in $\mathrm{H} \alpha$, the temperature has to be $\sim 8000 \mathrm{~K}$ or higher. Theoretical models of heating have to be able to explain these constraints. 


\section{Summary and Conclusions}

The present observational evidence suggests that:

- The winds of TTS have their origin in the disk, in a region exterior to the magnetosphere. They are powered by accretion.

- The indicators of the wind - forbidden lines and blueshifted absorption components in permitted emission lines - give consistent results for the mass loss rate: for most TTS, the winds are weak, $\dot{M}_{w} \leq 10^{-9} \mathrm{M}_{\odot} \mathrm{yr}^{-1}$. The mass loss rate increases with the rate of accretion in the disk, approximately as $\dot{M}_{w} / \dot{M} \sim 0.1$. The most massive winds have $\dot{M}_{w} \sim 10^{-7} \mathrm{M}_{\odot} \mathrm{yr}^{-1}$.

- Analyses of the blueshifted absorption component of permitted emission lines indicate that the temperature in the inner regions of the wind decreases as $\dot{M}_{w}$ increases. The temperature must be $\sim 10^{4} \mathrm{~K}$ for the low $\dot{M}_{w}$ objects, but less than $6000 \mathrm{~K}$ for $\dot{M}_{w} \sim 10^{-7} \mathrm{M}_{\odot} \mathrm{yr}^{-1}$.

- Although all CTTS have winds insofar as they have a disk, the winds of most of them are too tenuous to produce spatially extended observable jets.

Acknowledgments. I would like to thank the French Ministry of Foreign Affairs for a grant to pay my travel expenses to this conference. I also would like to thank the organizers for their support, L. Hartmann for helpful discussions and for figures 1 and 5, J. Najita for the profile of WL 16, and E. Gullbring for useful comments. This work was supported by NASA Grant 2306.

\section{References}

Appenzeller, I., Jankovics, I., Ostreicher, R.: 1984, AA, 141, 108

Basri, G., Marcy, G. W., Valenti, J. A.: 1992, ApJ, 390, 622

Blandford, R.D., Payne, D.G.: 1982, MNRAS 199, 883

Bertout, C., Basri, G., Bouvier, J.: 1988, ApJ, 330, 350

Bertout, C., Regev, O.: 1992, ApJ, 399, L163

Bacciotti, F., Hirth, G. A., Natta, A.: 1996, AA, 310, 309

Cabrit, S., Edwards, S., Strom, S. E., Strom, K. M.: 1990, ApJ, 354, 687

Calvet, N., Hartmann, L.: 1992, ApJ, 386, 239

Calvet, N., Hartmann, L., Hewett, R.: 1992, ApJ, 386, 229

Calvet, N., Magris C.,G., Patiño, A., D'Alessio, P.: 1992, Rev. Mexicana AA, 24, 27

Calvet, N., Hartmann, L., Kenyon, S. J.: 1993, ApJ, 402, 623

Camenzind. M.: 1990, in Rev. Mod. Astron. 3, ed. G. Klare, Sringer-Verlag, 234

Croswell, K., Hartmann, L., Avrett, E. H.: 1987, ApJ, 312, 227

Edwards, S., Cabrit, S., Strom, S., Heyer, I., Strom, K. M., Anderson, E.: 1987, ApJ, 321,473

Edwards, S., Hartigan, P., Ghandour, L., Andrulis, C.: 1994, AJ, 108, 1056

Folha, D., Emerson, J., Calvet, N.: 1997, Poster Proceedings of the IAU Symposium 182, eds. F. Malbet and A. Castets, Observatoire de Grenoble, 272

Giampapa, M. S., Basri, G., Johns, C. M., Imhoff, C. L. : 1993, ApJS, 89, 321

Giovanardi, C., Gennari, S., Natta, A., Ruggero, S.: 1991, ApJ, 367, 173

Ghosh, P., Lamb, F. K.: 1978, ApJ, 223, 83 
Gómez de Castro, A. I., Pudritz, R. E.: 1993, ApJ, 409, 748

Gullbring, E., Hartmann, L., Briceño, C., Calvet, N.: 1997, in preparation

Hamann, F.: 1994, ApJS, 93, 485

Hartmann, L., Edwards, S., Avrett, E.: 1982, ApJ, 261, 279

Hartmann, L., Raymond, J. C.: 1989, ApJ, 337, 903

Hartmann, L., Calvet, N., Avrett, E. H., Loeser, R.: 1990, ApJ, 349, 168

Hartmann, L., Hewett, R., Calvet, N.: 1994, ApJ, 426, 669

Hartmann, L., Kenyon, S. J.: 1996, ARAA, 34, 207

Hartmann, L., Calvet, N.: 1995, AJ., 109, 1846

Hartmann, L.: 1997, Accretion Processes in Star Formation, Cambridge University Press

Hartigan, P., Edwards, S., Ghandour, L.: 1995, ApJ, 452, 736 (HEG)

Hirth, G. A., Mundt, R., Solf, J.: 1993, AA, 285, 929

Hirth, G. A., Mundt, R., Solf, J., Ray, T: 1994, ApJ, L99

Holzer, E., Fla, T., Leer, E.: 1983, ApJ, 275, 808

Jankovics, I., Appenzeller, I., Krautter, J. : 1983, PASP, 95, 883

Johns, C. M., Basri, G.: 1995a, ApJ, 449, 341

Johns, C. M., Basri, G.: 1995b, AJ, 109, 2800

Johns-Krull, C. M., Basri, G.: 1997, ApJ, 474, 433

Kenyon, S. J., Hartmann, L.: 1987, ApJ, 323, 714

Kepner, J., Hartigan, P., Yang, C., Strom, S.: 1993, ApJ, 415, L119

Krautter, J., Appenzeller, I., Jankovics, I.: 1990, AA, 236, 416

Königl, A.: 1989, ApJ, 342, 208

Königl, A.: 1991, ApJ, 370, 39

Kuhi, L. V.: 1964, ApJ, 140, 1409

Kwan, J., Tademaru, E.: 1988, ApJ, 332, L41

Kwan, J., Tademaru, E.: 1995, ApJ, 454, 382

Lago, M. T., V. T.: 1984, MNRAS, 210, 323

Lago, M. T., V. T.: 1986, MNRAS, 222, 213

Lynden-Bell, D., and Pringle, J. E.: 1974, MNRAS, 168, 603

Mitskevich, A. S., Natta, A., Grinin, V. P.: 1993, ApJ, 404, 751

Muzerolle, J., Calvet, N., Hartmann, L.: 1997, in preparation

Najita, J. R., Shu, F. H.: 1994, ApJ, 429, 808

Najita, J., Carr, J. S., Tokunaga, A. T.: 1996, ApJ, 456, 292

Natta, A., Giovanardi, C.: 1990, ApJ, 356, 646

Natta, A., Giovanardi, C., Palla, F.: 1988, ApJ, 332, 921

Natta, A.: 1996, in ASP Conf. Proc. 109, Cool Stars, Stellar Systems and the Sun, eds. R. Pallavicini and A. Dupree, (San Francisco: ASP), 471

Ouyed, R., Pudritz, R. E.: 1994, ApJ, 423, 753

Paatz, G., Camenzind, M.: 1996, AA, 308, 77

Pelletier, G., Pudritz, R.E.: 1992, ApJ, 394, 117

Petrov, P. P., Herbig, G. H.: 1992, ApJ, 392, 209

Pringle, J. E.: 1989, MNRAS, 236, 107

Pudritz, R.E., Norman, C.A.: 1983, ApJ, 274, 677

Raga, A.: 1989, AJ, 98, 976

Safier, P. N.: 1993, ApJ, 408, 148

Shu, F. H., Najita, J. R., Ruden, S. P., Lizano, S.: 1994, ApJ, 429, 797

Shu, F., Najita, J., Ostriker, E.C., Shang, H.: 1995, ApJ, 455, L155

Solf, J., Böhm, K. H.: 1993, ApJ, L31 